\section{Advances in immunotherapy for melanoma}

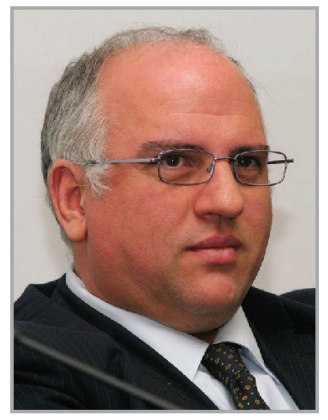

Paolo A Ascierto': Paolo A Ascierto obtained his MD from the University of Naples, Italy, where he earned board certification in oncology. He is currently Director at the Unit of Melanoma, Cancer Immunotherapy and Innovative Therapy Unit, National Tumor Institute 'Fondazione G. Pascale', Naples, Italy. Before his present position, he served consecutive positions there as a postdoctoral fellow and then as Vice Director of the Department of Clinical Immunology. His major research interests have included genetics and proteomics research of melanoma, apoptosis and cell death in human cancer and the assessment of molecular markers for tumor progression in melanoma, as well as the management of targeted therapies for melanoma, vaccination treatments and biochemical and immunological monitoring. He has served as a principal investigator in numerous clinical trials and has been widely published in peer-reviewed journals on topics related to his interests. Ascierto has been an invited speaker at more than 200 national and international meetings and is an active member of the Italian Society of Medical Oncology (AIOM), the American Society of Clinical Oncology (ASCO), the European Society of Medical Oncology (ESMO), the European Organisation for Research and Treatment of Cancer (EORTC) and the International Society for Biological Therapy of Cancer (iSBTc). He is also the Editor-in-Chief of the Combination Strategies section of the Journal of Translational Medicine and serves as a scientific reviewer for several journals.

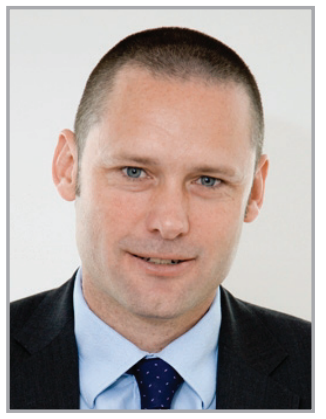

James Larkin*,2: James Larkin is a consultant medical oncologist specializing in the treatment of patients with cancer of the kidney and cancers of the skin, including melanoma. Larkin obtained a first-class degree in Natural Sciences from Cambridge University and undertook clinical training at Oxford University, qualifying in 1996. He underwent general medical training in London and, in 2001, won a Medical Research Council Fellowship for a Clinician, carrying out laboratory research at the Institute of Cancer Research (ICR), which led to the awarding of his PhD. He completed specialist training at The Royal Marsden Hospital and was appointed as a consultant in 2008. His research interests include the individualization of patient treatment in renal cancer and melanoma and the combination of novel targeted therapies for the treatment these diseases. He is the UK Chief Investigator for a number of clinical trials in melanoma and kidney cancer and has been awarded research grants from bodies including Cancer Research UK, the Wellcome Trust and the European Framework Programme 7. He is a member of the National Cancer Research Institute (NCRI) Melanoma Clinical Studies Group and Chair of both the NCRI Renal Cancer Clinical Studies Group and The Royal Marsden/ICR Committee for Clinical Research.

'Melanoma, Cancer Immunotherapy \& Innovative Therapy Unit, Istituto Nazionale Tumori Fondazione 'G. Pascale', Naples, Italy

${ }^{2}$ Royal Marsden Hospital, Fulham Road, London, SW3 6JJ, UK

*Author for correspondence: james.larkin@rmh.nhs.uk

Future Medicine ${ }_{\text {part of }}$ 
Q What impact has the approval of ipilimumab had on the prognosis of metastatic melanoma? Ascierto

After more than 30 years of failure in studies of advanced melanoma, ipilimumab was the first therapy to show a survival benefit in these patients. The pivotal MDX010-020 study reported a median overall survival (OS) of 10.0 months with ipilimumab compared with 6.4 months in the control arm, with 1-year and 2-year OS rates of 45.6 and $23.5 \%$, respectively. If we consider that, historically, patients with advanced melanoma typically had a median OS of approximately 6.2 months and that only $25 \%$ were alive at 1 year, the impact of ipilimumab on the prognosis of metastatic melanoma is clear.

Moreover, as was recently reported at the European Cancer Congress 2013, treatment with ipilimumab is able to render the disease as chronic in approximately $20 \%$ of patients. In a meta-analysis including 4846 patients who were treated with ipilimumab as part of a clinical trial or expanded-access program (EAP), 20\% of patients were still alive after 10 years.

Of course, there are still open questions that need to be addressed, such as the optimal dosage (3 vs $10 \mathrm{mg}$ ) and whether maintenance treatment is useful or not. Ongoing studies will hopefully provide answers to these questions.

Larkin

Ipilimumab was approved for use in 2011 and, for the first time, we were able to offer patients a generally well-tolerated outpatient treatment that had the prospect in some patients of controlling advanced disease for a number of years. Approximately $15-20 \%$ of patients probably receive long-term benefit from therapy, and recent data presented at the European Cancer Congress conference in Amsterdam, The Netherlands, in October 2013 showed that for those receiving treatment with ipilimumab, if they were alive 3 years after treatment, the chances are that they would be alive at 5 years and probably beyond this. Ipilimumab has had a big impact on the treatment of this disease, although of course, a major aim in the future will be to increase the number of patients who are benefitting from treatment with immunotherapy.

\section{Q What do we currently know about using ipilimumab as a combination therapy?}

Ascierto

Combination therapies represent the future of melanoma treatment, and this is especially true if we consider ipilimumab. Although the initially interesting combination of ipilimumab plus the glycoprotein 100 melanoma peptide vaccine (gp100) did not improve on ipilimumab alone, Stephen Hodi of the Dana-Farber Cancer Institute (MA, USA) reported promising data on the combination of ipilimumab with bevacizumab at the American Society of Clinical Oncology (ASCO) 2011 annual meeting. More recently, data regarding ipilimumab in combination with GM-CSF were reported at ASCO 2013, while even more promising are the data on the combination of ipilimumab with nivolumab (an anti-PD1 agent), which showed an overall response rate (ORR) of $53 \%$, with $41 \%$ of responses involving a tumor shrinkage of more than $80 \%$. Unfortunately, the ability to combine ipilimumab with the BRAF inhibitor vemurafenib failed because of increased liver toxicity. However, a study of ipilimumab in combination with dabrafenib/trametinib is ongoing. Other trials are also exploring interesting combination therapies, such as ipilimumab or nivolumab with newer compounds including urelumab (an anti-CD137), anti-killer cell immunoglobulin-like receptor (ClinicalTrials.gov identifier: NCT01750580), anti-lymphocyte activation gene-3 (NCT01968109), IL-21 (NCT01489059) and anti-PD-L1 with dabrafenib/trametinib (NCT02027961).

Larkin

In randomized trials, ipilimumab was combined with dacarbazine and also with a vaccine, but the consensus is that neither of these early combinations enhance the efficacy of ipilimumab, and so the current licensed schedule of the drug is as a single agent of $3 \mathrm{mg} / \mathrm{kg}$. A number of combinations of ipilimumab are currently being investigated in clinical trials. One example is the combination of ipilimumab with the anti-VEGF agent bevacizumab, and a further combination that has generated a lot of excitement is with the anti-PD1 agent nivolumab. The data published in the middle of 2013 in the New England Journal of Medicine and presented at ASCO 2013 suggested that the concurrent administration of these drugs could lead to dramatic responses in many patients, but this combination needs to be tested in randomized trials, which are currently ongoing.

Q Do you think ipilimumab will show benefits for rarer melanomas? If so, which ones?

\section{Ascierto}

Although most melanomas occur on the skin surface, a small percentage are noncutaneous, 
occurring most often in the eye and mucous membranes. Clinical trial data are scarce in these patients due to the rarity of these disease subtypes.

The good news is that the ipilimumab data suggest that it is effective across all patient populations. In fact, results from the US and Italian EAPs show 1-year OS rates of 34 and 32\%, respectively, for uveal melanoma, while similar 1 -year survival rates of 32 and $39 \%$, respectively, were seen in patients with mucosal melanoma. These are consistent with the 1-year survival rates in the general EAP populations. Moreover, at this time, ipilimumab is the only available treatment with favorable efficacy and safety results in patients with these rare melanomas.

\section{Larkin}

There are no prospective data for treating rare melanomas (i.e., mucosal and uveal melanomas) with ipilimumab, and so we have to rely on retrospective case series. Randomized trials in these rare subsets of melanoma are very difficult to conduct. Nevertheless, there is some evidence that ipilimumab may have some activity in uveal melanoma, and this comes from a subset of patients in the Italian EAP and also some series from other institutions, including The Royal Marsden Hospital. Therefore, I think that ipilimumab is a reasonable treatment to consider outside the context of a clinical trial in a patient with uveal melanoma. In terms of mucosal melanomas, there has been at least one case report of efficacy, so again, I think this is something worth considering outside of trials. However, I think the most important issue is that all patients with melanoma, particularly patients with rare melanoma, should have the opportunity to participate in a clinical trial.

\section{Q What is the latest on the use of IL-2 for metastatic melanoma?}

\section{Ascierto}

There are no recent updates on the use of IL-2 for metastatic melanoma of great clinical importance. Some researchers have reported that the effect of vemurafenib is increased in patients who are pretreated with IL-2, and it was also observed that IL-2 seems to result in longer progression-free survival and OS in patients with the NRAS mutation versus wild-type patients. However, these observations need to be verified in clinical studies. Despite a recent expert panel considering IL-2 as the first-line therapeutic option for specific subgroups of patients with good performance status, such treatment is not often used in Europe. In fact, high-dose IL-2 therapy is associated with frequent and severe toxicity with documented low activity, although some patients can remain disease free for several years.

Larkin

Historically, high-dose IL-2 has been used to treat advanced melanoma, but there has certainly never been much use of high-dose IL-2 in the UK. There are ongoing programs at some centers in the USA, and I think that, for appropriately selected patients, this may be something to consider. However, I suspect that the role of high-dose IL-2 will diminish with the approval of newer, less toxic immunotherapies, and again, I would stress that if treatment with high-dose IL-2 is being contemplated, this should ideally be taking place in the context of a clinical trial.

\section{Q How effective do you think high-dose} interferon is? In your opinion, have we moved on from this treatment option?

\section{Ascierto}

It was clearly reported in the latest meta-analysis in 2010 that the relative benefit of adjuvant treatment with interferon (IFN) is $18 \%$ for diseasefree survival and $11 \%$ for OS. This may be translated into an absolute benefit of $3 \%$, and this is regardless of dosage, route of administration (intramuscular, intravenous or subcutaneous) and the duration of treatment. For this reason, adjuvant treatment with IFN is justified in patients with melanoma. However, it is clear that this is a very limited benefit and so we must go beyond IFN. The first and crucial point is to identify possible predictive markers of the response to IFN. One such possible marker is ulceration of the primary lesion. As reported in the European Organisation for Research and Treatment of Cancer (EORTC) studies (EORTC18952 and EORTC18991), ulceration was associated with a better response to IFN in patients with stage IIBN1 melanoma, but not in patients with stage IIIN2 disease. An ongoing prospective clinical trial (EORTC18081) is further exploring this hypothesis by investigating the use of adjuvant peg-IFN$\alpha-2 b$ for 2 years compared with observation in patients with an ulcerated primary cutaneous melanoma with $\mathrm{T}(2-4) \mathrm{bN} 0 \mathrm{M} 0$. It will also be interesting to assess the possible combinations of IFN with new compounds, such as ipilimumab and vemurafenib/dabrafenib. For instance, preclinical data reported by Soldano Ferrone and 
colleagues at the Massachusetts General Hospital (MA, USA) have suggested that expression of the IFN receptor is downregulated in the presence of $B R A F$ mutations, but upregulated by vemurafenib administration, resulting in increased efficacy when vemurafenib and IFN are given in combination. Two clinical trials (NCT01943422 and NCT01959633) are currently exploring the activity of this combination in advanced disease. In addition, the combination of IFN with antiPD1 agents is of interest, since IFN has the ability to increase the expression of PD-L1 on melanoma cells.

Larkin

Adjuvant high-dose IFN remains a standard of care at some institutions, although this is not something that we recommend. It is noteworthy of the ongoing adjuvant trials at the moment that some have a high-dose IFN control arm and some have a placebo control arm, and particularly in the USA, high-dose IFN is certainly used more often than in the UK. There is no doubt that high-dose IFN reduces the risk of relapse from melanoma, but the effect on OS is relatively marginal and, of course, the treatment is toxic.

\section{Q What promise do you think anti-PD1 therapy holds?}

\section{Ascierto}

Phase I-II studies with anti-PD1 therapy have reported higher ORRs than those observed with ipilimumab, with earlier responses and an increase in the number of patients with longterm responses. For instance, in the nivolumab study (NCT00730639) reported at ASCO 2013, the ORR was $31 \%$, with a median duration of response of 2 years, while the 1 -year and 2-year OS rates were 62 and 43\%, respectively. It was also recently reported at the Society for Melanoma Research International Congress that the 1-year OS rate for the anti-PD1 MK3475 (previously known as lambrolizumab) was $82 \%$. My belief is that anti-PD1 therapy will increase the number of patients who achieve 'chronic disease' status. Considering that the 1-year OS rate was similar for MK3475 alone and the combination of nivolumab with ipilimumab, the results of Phase III trials are required in order to confirm whether single-agent treatment is superior to combination therapy.

\section{Larkin}

There are a number of anti-PD1 drugs in development, of which those furthest forward (at least in melanoma) are nivolumab, made by BristolMyers Squibb, and MK3475, made by Merck. There is no doubt from nonrandomized trials that these drugs have activity and appear to be very well tolerated. Randomized trials comparing these drugs with standards of care are currently recruiting, but I think it is highly likely that these drugs will be approved for the treatment of melanoma in the future, and I hope that they will have the possibility - similar to ipilimumab - of controlling melanoma for very long periods of time, but again, we need to investigate this in trials.

\section{Q There are several types of cancer vaccine} currently in trials that could, potentially, be used to bolster the immune system of a melanoma patient. Do you think that they will be successful?

\section{Ascierto}

After the recent failure of the adjuvant trial with the MAGE-A3 vaccine, which adds to several previous failures in this field, my opinion is that vaccination alone is not capable of changing the natural history of melanoma. Although it was previously shown that the addition of a gp100 vaccine is able to increase the efficacy of IL-2, studies with CancerVax ${ }^{\mathrm{TM}}$, vaccinia melanoma cell lysates, GM2-ganglioside and, finally, MAGE-A3 were all negative and, in some instances, the use of vaccination was actually detrimental. In addition, given that the combination of ipilimumab plus gp100 was not superior to ipilimumab alone, combining vaccines with other compounds such as antiPD1 agents will probably be a more beneficial approach. In fact, it has been reported that vaccination might increase the expression of PD1 on immune system cells, which may translate into tolerance against tumor cells. Another different approach for the future might be to use a vaccine in sequential therapy with the checkpoint blockade antibodies or as maintenance treatment after induction with more effective agents.

Larkin

To date, vaccine therapies, despite lots of effort, have not been successful in treating melanoma. Nevertheless, this is clearly an exciting field, but I believe that the role of vaccines in the future might be a little diminished if the antiPD1 drugs deliver as much as everyone hopes. However, I still think that this is a very important area of investigation. 
Q Immunotherapy options are known to have some fairly serious side effects - what are they and how can we minimize these? Ascierto

While vaccinations are mostly associated with simply local and nonsevere reactions, the immunomodulating antibodies can result in more serious immune-related adverse events (irAEs). The impact of these irAEs that are associated with ipilimumab treatment is well known, with the most important being skin reactions, colitis/diarrhea, liver toxicity and endocrinopathies. There are also minor toxicities, including neuropathies and hematological toxicities, which are rather infrequent. The percentage of patients with grade 3-4 irAEs with ipilimumab is dose dependent, but is approximately $10-15 \%$ with the approved $3 \mathrm{mg} / \mathrm{kg}$ dosage. However, although they can sometimes be life threatening, in general, these irAEs are well tolerated and manageable through the use of specifically developed treatment algorithms. What is most important is the early diagnosis and treatment with corticosteroids according to these treatment algorithms. Steroids drastically reduce the possible occurrence of severe consequences, such as the incidences of bowel perforation seen in the early phases of ipilimumab development. The anti-PD1 agents have a similar toxicity profile, with the same effects on the skin, GI tract, liver and endocrine system. However, the occurrence of grade 3-4 adverse events is lower than those seen with ipilimumab, affecting approximately $5-6 \%$ of patients treated with both nivolumab and MK3475. The risk of pneumonitis with the anti-PD1 treatments should also be mentioned; however, this was only observed as a grade 1-2 adverse event in 4 and $4.4 \%$ of patients in the nivolumab and MK3475 studies, respectively.

\section{Larkin}

With regards to ipilimumab, I say to patients that $80-90 \%$ of the time there are no serious side effects, but a minority of patients will have potentially significant side effects. These are as a consequence of the induction of autoimmunity and a range of side effects can occur. These include dermatitis, hepatitis, colitis, uveitis and hypophysitis, and there are a number of rarer side effects, including neurological syndrome such as Guillain-Barré syndrome. In my opinion, the most important thing about these side effects is counseling patients very carefully so that we are able to pick up on the side effects early. Side effects are normally manageable with the use of steroids, although it is occasionally necessary, particularly with colitis, to use other agents, such as anti-TNF therapy. Based on preliminary evidence, the anti-PD1 agents appear to have a similar spectrum of side effects to ipilimumab, although pneumonitis may be a bit more common. However, the anti-PD1 drugs seem to be very well tolerated overall.

\section{Q Should immunotherapy be initiated for all patients at the first metastatic relapse?}

\section{Ascierto}

My answer to this question is yes, for several reasons. First of all, as previously stated, just four injections of ipilimumab has the potential to render the disease as chronic in approximately $20 \%$ of patients. Moreover, it provides a survival benefit (which may range from a few months to a number of years) in approximately another $60 \%$ of patients. By contrast, the evidence at present suggests that almost all patients treated with targeted agents have disease recurrence, and there are no available data on long-term survival. Another important issue is that clinical trials and single-center experiences clearly show that approximately $40 \%$ of patients with disease recurrence after BRAF inhibitor treatment have rapid disease progression and die within 1-2 months. Because of this, these patients unfortunately do not have the opportunity to be treated with ipilimumab as a second-line treatment. On the other hand, ipilimumab treatment as the first choice does not select for fast progression, while BRAF inhibitors are equally effective in patients who are pretreated with ipilimumab. As such, ipilimumab should be used as the firstline therapy before a BRAF inhibitor. The exception to this is those patients with a performance status of $\geq 2$ and symptomatic brain metastases, who should be treated immediately with targeted agents, given their more immediate impact on disease.

In the future, results from studies of anti-PD1 agents and ipilimumab plus nivolumab combination treatment will help to confirm the role of immunotherapy.

Larkin

My view is that drugs such as ipilimumab, at least as single agents, work best in patients with low-volume disease. I want all of my patients to have the opportunity to be treated with immunotherapy at some stage. Therefore, I would tend to use immunotherapy if possible in most patients in the first-line setting, but for patients 
who have more rapidly progressive disease and a mutation in a gene such as $B R A F$, thereby predicting response to a targeted therapy, I would use targeted therapies.

\section{Q How close are we to finding predictive biomarkers for ipilimumab \& anti-PD1 agent response? \\ Ascierto}

We know that ipilimumab improves OS but not surrogate end points, such as ORR or progression-free survival. We also know that no specific subgroups of patients receiving an increased benefit from ipilimumab have been identified, and that the efficacy of ipilimumab is the same regardless of $B R A F / N R A S / c K i t$ mutational status. As such, biomarkers that can predict the patients who are most likely to benefit from ipilimumab therapy would be very welcome.

There is ongoing debate over whether increased absolute lymphocyte count after two ipilimumab treatments (week 7) correlates with clinical benefit and OS. The data are controversial and the findings need to be further assessed and validated in a prospective trial. In recent years, we have also observed other potential predictive markers, including the expression of the ICOS molecule, LDH and CRP levels, circulating Treg levels, pretreatment levels of myeloid-derived suppressor cells and the presence of a humoral immune response against the NY-ESO-1 tumor antigen. Recently, at the Society for Immunotherapy of Cancer (SITC) 2013 Annual Meeting, Laurence Zitvogel (Institut Gustave Roussy, France) reported interesting data on how high levels of soluble IL-2R- $\alpha$ combined with high LDH-5 levels predicted resistance to ipilimumab. All of these data testify to the significant efforts being made to identify predictive biomarkers for response to ipilimumab. However, at this stage, none of these biomarkers are close to being used in clinical practice.

Identifying predictive biomarkers to anti-PD1 treatment offers a different story, since it is logical that PD-L1 tumor expression might represent a good predictive marker of treatment efficacy. However, the presence of approximately $20 \%$ of patients who are PD-L1 negative but respond to anti-PD1 therapy complicates matters. Moreover, not all patients who express PD-L1 respond to anti-PD1 treatment (between 36 and $44 \%$ in different reports). This finding should of course be investigated in order to verify whether it is due to technical issues (e.g., the cutoff for PD-L1 positivity, use of the correct antibody, staining procedures and topographic localization of the PD-L1-positive cells, among other factors) or, very probably, unknown factors. For instance, the presence of PD-L1 in the tumor might not necessarily be related to the tumor invasion, but may instead be due to the antitumor immune response, with the production of IFN stimulating the expression of PD-L1. Thus, for anti-PD1 therapy, PD-L1 expression may be a good predictive biomarker, but is probably not sufficient alone, and more research is needed.

Future new approaches will include the gene signature approach, the immunoscore/immunoprofiling approach and the functional assay of immune signaling in peripheral blood mononuclear cells (single-cell network profiling), all of which could help in patient selection for treatment with immunomodulating antibodies. Larkin

This is a critical area. There has been a lot of work on ipilimumab over the years, but nothing has been found that we can use reliably at baseline in order to say whether a patient will respond to therapy or not, although my own opinion is that the patients who have the highest chance of benefit are those who do not have high-tempo disease. This is an enormous area of ongoing interest and I suspect in the next few years we will develop predictive biomarkers for these drugs, but I think this may be complex given the mechanisms of action of these drugs, and maybe we should think about predictive profiles, rather than one particular factor that will predict response.

Financial \& competing interests disclosure PA Ascierto has had a consultantladvisory role for BristolMyers Squibb, Roche-Genentech, Merck Sharp \& Dohme, GlaxoSmithKline (GSK), Ventana and Novartis. He has received research funds from Bristol Myers Squibb, RocheGenentech, Merck Sharp \& Dohme, and Ventana, and has also received honoraria from Bristol-Myers Squibb, RocheGenentech and GSK.J Larkin has received research funding Novartis and Pfizer, and consultancy (non-remunerated since 2012) fees from Pfizer, Novartis, BMS, GSK, MSD and Roche-Genentech. The authors have no other relevant affiliations or financial involvement with any organization or entity with a financial interest in or financial conflict with the subject matter or materials discussed in the manuscript apart from those disclosed.

No writing assistance was utilized in the production of this manuscript. 